\title{
Preliminary examination of the comet Wild 2 samples returned by the Stardust spacecraft
}

\author{
Michael E. Zolensky ${ }^{1}$, Donald E. Brownlee ${ }^{2}$, Peter Tsou ${ }^{3}$, \\ Friedrich P. Hörz ${ }^{1}$, Scott A. Sandford ${ }^{4}$, George J. Flynn ${ }^{5}$, \\ Kevin D. McKeegan ${ }^{6}$, and Lindsay P. Keller ${ }^{1}$ \\ ${ }^{1}$ NASA Johnson Space Center, Houston, TX 77058, USA \\ email: michael.e.zolensky@nasa.gov \\ ${ }^{2}$ Department of Astronomy, University of Washington, Seattle, WA 98195, USA \\ ${ }^{3}$ Jet Propulsion Laboratory, CalTech, Pasadena, CA 91109-8099, USA \\ ${ }^{4}$ NASA Ames Research Center, Moffett Field, CA 94035, USA \\ ${ }^{5}$ Dept. of Physics, State University of New York at Plattsburgh, Plattsburgh, NY 12901, USA \\ ${ }^{6}$ Dept. of Earth and Space Sciences, University of California, Los Angeles, CA 90095, USA
}

Abstract. Results of the Stardust mission to sample dust from comet Wild 2 are summarized.

Keywords. comets, comet Wild 2, space vehicles

\section{Results}

The sample return capsule of the Stardust spacecraft was successfully recovered in northern Utah, USA, on January 15, 2006, and its cargo of coma grains from comet Wild 2 has now been the subject of intense investigation.

The period since spacecraft recovery been sufficient to permit numerous analyses by over 200 researchers to have been performed and to permit some understanding of the following fundamental sample issues:

(1) Comet nucleus composition, mineralogy, petrology, isotopic composition and grain physical properties.

(2) Sample variability.

(3) Type and degree of sample alteration by the collection process, and subsequent sample handling.

(4) Sample documentation and handling procedures.

(5) Comparisons to the Deep Impact mission to comet Tempel I.

Following the close of sample preliminary examination, Stardust samples will be made available to the larger community, as are lunar samples, IDPs, and Antarctic meteorites. A sample catalog will be available at the NASA Johnson Space Center Curation website. A dedicated peer review committee will consider all sample requests. The Stardust interstellar tray is being scanned in the Cosmic Dust Lab; when this operation is complete (approximately by the end of 2006) the Cosmic Dust Lab will be re-opened for business.

\section{Mission details}

Launch date: 7 February 1999

Arrival at comet Wild 2: 2 January 2004

Return to Earth: 15 January 2006 
Payload mass: $270 \mathrm{~kg}$.

Primary science instruments:

Stardust-SRC (sample Return Capsule) and Aerogel Collector: a compact system, consisting primarily of a sample canister with an aeroshield/basecover, plus navigation recovery aids, an event sequencer and a small parachute system.

Stardust-CIDA (Cometary and Interstellar Dust Analyzer): intercepts dust and performs real-time compositional analysis for transmission back to Earth.

Stardust-NC (Navigation Camera): used to navigate the Stardust spacecraft upon approach to the comet, the camera also served as an imaging camera to capture high-resolution color images of the comet nucleus on approach and on departure, and broadband images at various phase angles while nearby.

Stardust-DFMI (Dust Flux Monitor Instrument): records the impact of small particles; in addition, two separate acoustic impact sensors monitor strikes by larger particles.

\section{In the literature}

A detailed description of the Stardust mission encounter with comet Wild 2 was published in the 18 June 2004 issue of Science. Brownlee et al. (2004) describe the surface of comet $81 \mathrm{P} /$ Wild 2 as seen from the Stardust spacecraft. Sekanina et al. (2004) modeled the nucleus and jets of the comet based on data from the Stardust encounter. Kissel et al. (2004) discuss results from the cometary and interstellar dust analyzer during the encounter. And Tuzzolino et al. (2004) report on the dust Measurements in the comet's coma as measured by the dust flux monitor instrument.

The first reports of analysis from the returned samples has to date been presented in special sessions of the Meteoritical Society and the American Geophysical Union annual meetings. A full refereed report is in preparation.

\section{References}

Brownlee, D. E., Horz, F., Newburn, R. L., Zolensky, M., Duxbury, T. C., Sandford, S., Sekanina, Z., Tsou, P., Hanner, M. S., Clark, B. C., Green, S. F., \& Kissel, J. 2004, Science, 304, 1764

Kissel, J., Krueger, F. R., Silén, J., \& Clark, B. C. 2004, Science, 304, 1774

Sekanina, Z., Brownlee, D. E., Economou, T. E., Tuzzolino, A. J., \& Green, S. F. 2004, Science, 304,1769

Tuzzolino, A. J., Economou, T. E., Clark, B. C., Tsou, P., Brownlee, D. E., Green, S. F., McDonnell, J. A. M., McBride, N., \& Colwell, M. T. S. H. 2004, Science, 304, 1776 